\title{
Reflexión epistemológica para la investigación de los procesos de determinación social de la salud
}

\author{
Epistemological reflection for the investigation of the processes of social \\ health determination
}

\section{Reflexão epistemológica para a investigação dos processos de determinação da saúde social}

Juan Paulo Ruiz Taborda'; Luis Felipe Higuita Gutiérrez²; Jaiberth Antonio Cardona Arias ${ }^{3}$

1 Microbiólogo y bioanalista, MSc (estudiante) Salud Pública. Grupo de Investigación Salud y Sostenibilidad, Universidad de Antioquia. Medellín, Colombia. ORCID: https://orcid.org/0000-0001-7820-6015.

2 Microbiólogo y bioanalista, MSc Educación y Desarrollo Humana, PhD (candidato) Ciencias Sociales. Escuela de Microbiología, de la Universidad de Antioquia, Facultad de Medicina Universidad Cooperativa de Colombia. Medellín, Colombia. ORCID: https://orcid. org/0000-0003-1361-3124.

3 Microbiólogo y bioanalista, MSc Epidemiología, MSc Economía aplicada, PhD (candidato) Salud Pública. Profesor Escuela de Microbiología, Universidad de Antioquia. Medellín, Colombia. ORCID: https://orcid.org/0000-0002-7101-929X

Recibido: 30/04/2020. Aprobado: 27/10/2020 . Publicado: 27/01/2021

Ruíz-Taborda JP, Higuita-Gutiérrez LF, Cardona-Arias JA. Reflexión epistemológica para la investigación de los procesos de determinación social de la salud. Rev. Fac. Nac. Salud Pública. 2020;39(1):e341437. Dor: https://doi. org/10.17533/udea.rfnsp.e341437

\section{Resumen}

Un debate central en la salud pública contemporánea gira en torno de los determinantes sociales de la salud, en las posturas de la Organización Mundial de la Salud y de la medicina social latinoamericana. Dado que, en la literatura sanitaria, se presentan múltiples críticas al modelo de dicha organización, así como a su énfasis positivista, a su reduccionismo de la ontología de lo social y a la linealidad de sus métodos, al tiempo que existen pocos textos en salud sobre el "realismo crítico" como fundamento epistemológico de la corriente latinoamericana, el presente trabajo tiene como objetivo describir algunos hitos históricos de la determinación social de la salud y las características del realismo crítico como principal postura epistemológica para esta corriente. Desde esta postura se evidencia una suerte de "ingenuidad" epistemológica en el modelo de la Organización Mundial de la Salud; también demuestra que la diferencia de ambos enfoques no es reductible a una semántica o estilo de abordaje de lo social en salud, y pone de manifiesto retos operativos para la corriente latinoamericana.

--------Palabras clave: Medicina social, epidemiología social, determinantes sociales de la salud, epistemología, realismo crítico, Organización Mundial de la Salud. 


\section{Abstract}

A central debate in contemporary public health revolves around the social determinants of health in the model of the World Health Organization (wHO) and Latin American social medicine. Considering that in the health literature there are multiple criticisms of the WHO approach such as its positivist emphasis, reductionism of the ontology of the social and linearity of its methods; at the same time that there is a low amount of health texts about "critical realism" as the epistemological foundation of the Latin American current; The present work exposes some historical concepts of the
Latin American proposal and tries to deepen the development of its epistemological positioning from the "critical realism". Critical realism demonstrates a sort of epistemological "naivety" in the WHO model, demonstrates that the difference of both approaches is not reducible to a semantics or social approach of the health, and highlights operational challenges for the model Latin American

-Keywords: Social Medicine; Social Epidemiology; Social Determinants of Health; Epistemology; Critical Theory

\section{Resumo}

Um debate central em saúde pública contemporânea gira em torno dos determinantes sociais da saúde nas posições da Organização Mundial da Saúde (OMS) e da medicina social latino-americana. Considerando que na literatura em saúde existem múltiplas críticas à corrente da OMS, como ênfase positivista, reducionismo da ontologia do social e linearidade de seus métodos; embora exista uma baixa quantidade de textos sobre saúde sobre "realismo crítico" como fundamento epistemológico da corrente latino-americana; O presente trabalho expõe alguns conceitos históricos da proposta latino- americana e tenta aprofundar o desenvolvimento de seu posicionamento epistemológico a partir do "realismo crítico". O "realismo crítico" evidencia uma espécie de "ingenuidade" epistemológica no modelo da oms, demonstra que a diferença entre as duas abordagens não é redutível a uma semântica ou abordagem à saúde social e destaca os desafios operacionais para a modelo América Latina

-Palavras-chave: Medicina social; Epidemiologia Social; Determinantes sociais da saúde; Epistemologia; Teoria Crítica

\section{Introducción}

En el inicio de este siglo, la salud pública mundial ha recabado evidencia científica y política sobre la necesidad y la urgencia de orientar las acciones sanitarias hacia la disminución de las desigualdades en salud e incrementar la equidad al respecto. En este marco se creó, en 2005, la Comisión de Determinantes Sociales de la Salud, de la Organización Mundial de la Salud (oMs), cuyos principios también fueron asumidos por la Organización Panamericana de la Salud (oPs), haciendo un llamado a los gobiernos para tomar medidas que impacten los determinantes socioeconómicos, políticos y culturales de la salud [1], los cuales son definidos como "las circunstancias en que las personas nacen, crecen, viven, trabajan y envejecen, incluido el sistema de salud", circunstancias que reflejan la distribución del capital económico, el poder y los recursos materiales en los ámbitos mundial, nacional y local [2]. Con los determinantes sociales, se amplía el análisis de los problemas de salud con dimensiones que influyen en la cotidianidad de las personas y trascienden los sistemas de salud, como lo son las "po- líticas y sistemas económicos, programas de desarrollo, normas y políticas sociales y sistemas políticos" que explican las desigualdades e inequidades sanitarias [2,3].

Dados los vínculos entre los determinantes sociales y la salud, estos hacen parte de los "Objetivos de desarrollo del milenio" de las Naciones Unidas [4] y de diversas políticas para superar las inequidades sanitarias, las cuales constituyen la principal barrera para el desarrollo. Uno de esos objetivos es la estrategia de "acceso universal a la salud y la cobertura universal de salud", que propende por el mejoramiento del desarrollo socioeconómico y la atención en salud $[3,5]$.

Los determinantes sociales de la salud de la oms y la OPS se agrupan en una estructura que incluye el contexto socioeconómico y político (gobernanza; política macroeconómica, sanitaria y social; normas y valores culturales), determinantes estructurales (posición social, dada por educación, ocupación, ingresos, género y etnia), determinantes intermediarios (condiciones materiales de vida y cohesión social), las conductas individuales, 
psicosociales y genéticas, y la prestación de servicios de salud, bajo una concepción de la salud como un bien público [1,3,5-7].

Pese a las pretensiones de novedad de la oms y la ops con su modelo de determinantes sociales, ya desde la década de los setenta, en Latinoamérica, se había acuñado la "determinación social de la salud" (DSS) como una categoría central de la medicina social y la epidemiología crítica que, como otras posturas críticas, no tuvo desarrollo amplio en el sector sanitario, por el predominio y la hegemonía de la epidemiología anglosajona y la salud pública tradicional (burocratizada, positivista, biomédica). En tal sentido, la consolidación de la Comisión de Determinantes Sociales de la Salud en 2005 fungió como condición de posibilidad para una suerte de reapertura o fortalecimiento de discusiones en salud pública que, en el caso latinoamericano, reiteran la importancia de las visiones críticas al modelo de la OMS-OPS, a la escuela tradicional de la salud pública y a algunos organismos internacionales relacionados con el sector salud $[8,9]$.

Las diferencias entre el modelo de la DSs latinoamericano y el de la OMS-OPS va más allá de un tema semántico, dado que el primero alude una serie de categorías trabajadas en corrientes críticas al neoliberalismo y al predominio de las lógicas emanadas de la epidemiología clásica [8]. En este sentido, Jaime Breilh expone estas diferencias y señala la ruptura que representa la DSs respecto a la epidemiología tradicional, que incluye el modelo de la oms-OPs $[9,10]$. Otros autores han detallado las diferencias conceptuales, éticas y políticas, y han resaltado las divergencias sobre la causalidad, el riesgo y la concepción del proceso salud-enfermedad $[11,12]$.

En medio de este debate, los ejes más controvertidos han girado en torno a las esferas política y metodológica, mientras que la fundamentación epistemológica del modelo latinoamericano resulta menos problematizada (las mayores tensiones se han endilgado a su compromiso político crítico y su bajo desarrollo metodológico). En este sentido, la pregunta orientadora de este ensayo es: ¿cuáles son los principales elementos epistemológicos para la investigación en Dss? El objetivo es describir algunos hitos históricos de la DSs y las características del realismo crítico, como principal postura epistemológica para esta corriente. Para ello, se realizó una selección de artículos teóricos recientes sobre DSS, mediante cuatro estrategias de búsqueda y usando filtros específicos de la fuente: en SciElo [(ti:(determinación social de la salud)) y (ti:(realismo crítico))] y en Google Scholar [(allintitle: determinación social de la salud) y (allintitle: realismo crítico)], con una selección de textos por conveniencia para exponer hitos históricos de la propuesta de Dss y su posicionamiento epistemológico desde el realismo crítico.
Es oportuno aclarar que, en este ensayo, no partimos de la filosofía que fundamenta al realismo crítico, sino de cómo esta postura aparece en la producción académica sobre DSs y, en consecuencia, este texto se configura como un proceso descriptivo y de reflexión de los fundamentos epistemológicos de la Dss latinoamericana, los cuales servirán para su consolidación conceptual, metodológica y práctica.

\section{Algunos antecedentes histórico-políticos}

La DSS es una categoría que nace en el seno de la epidemiología crítica latinoamericana, como un proceso de ruptura con el multicausalismo lineal y el modelo empírico-funcionalista de la epidemiología clásica y ecológica. Las fuentes teóricas que sirvieron a la génesis de la epidemiología crítica se cimientan en planteamientos revolucionarios en el campo sanitario y su determinación sociohistórica en los siglos XVIII y XIX, con las luchas anticoloniales y obras revolucionarias de Europa, las cuales desarrolla Howard Waitzkin en Medicina y salud pública al final del imperio $[9,10]$.

En este devenir histórico se materializan, a grandes rasgos, dos características de la DSs: la primera es el carácter antiimperialista y de identidad latinoamericana, que trae como referentes y pioneros a Eugenio Espejo y Manuela Espejo, con sus aportes teóricos y prácticos a la salud del movimiento independentista bolivariano $[9,10]$. La segunda es el análisis de las fuerzas económicas y políticas que determinan el desarrollo de la medicina corporativa, en el que se señala el fracaso de las estructuras políticas tradicionales, al tiempo que se cuestiona el hecho que las políticas públicas sean dictadas por un Estado transnacional no elegido o por intereses corporativos, cristalizados en la Organización Mundial de Comercio, el Banco Mundial y otras instituciones $[9,10,13]$.

A lo anterior se sumó un contexto políticamente convulsionado en América Latina en la segunda mitad del siglo xx, con fenómenos sociales como la Guerra Fría, que se relacionaron con procesos revolucionarios en Latinoamérica, especialmente la Revolución cubana de 1959, que marcó un punto de quiebre para la constitución de los principios de la salud pública socialista, orientados por un proyecto político de transformación de la salud poblacional. En el caso cubano, se dio prioridad al sector de la salud, mediante la intervención de problemas estructurales de tipo político y socioeconómico, con políticas que derivaron en algunos logros, como el aumento de camas hospitalarias (28 536 en el año 1958 y 41019 en 1968), la ampliación de servicios de salud en áreas alejadas a la capital, el énfasis en la atención primaria, la creación del servicio médico social rural, la gratuidad de la atención, la disminución de previos 
de medicamentos, la nacionalización de los laboratorios farmacéuticos, entre otros [14].

Paralelamente, en Chile, el trabajo del presidente Salvador Allende, influenciado por Eduardo CruzCoke, ${ }^{*}$ permitió la construcción de los cimientos de la medicina social chilena. Durante su ejercicio de la presidencia en 1970, promovió cambios importantes, como la vinculación de los obreros a las decisiones de gobierno, la ampliación de la cobertura de la atención en salud y la creación de los comités paritarios de higiene y salud industrial. Es importante señalar que los resultados de estas medidas no pudieron ser evaluados, dado el golpe de Estado, la muerte de Allende y la consecuente ausencia de los representantes de este modelo de salud pública en Alma Ata ${ }^{\dagger}[16,17]$.

Debido a lo anterior, el pensamiento social revolucionario penetró la salud pública y favoreció la aparición de otras figuras en las posturas críticas en la epidemiología, como el médico Ricardo Paredes, quien luego de estudiar los aspectos sociales, ocupacionales y sanitarios de un sector minero, publicó su trabajo sobre la DSs en la minería imperial, presentando un análisis sociológico y epidemiológico de menoscabo de la salud y el medio ambiente ecuatoriano [9,10].

Además, a principios de la década de los setenta, en los núcleos investigativos derivados de estas posturas, se concibió la epidemiología crítica como uno de los ejes disciplinares de la medicina social latinoamericana y se comenzó a hablar de manera explícita de la DSs, con lo que se retomó la discusión de la relación entre el sistema social imperante y la salud de las poblaciones, mediante categorías del pensamiento marxiano, como "sistema económico", "trabajo", "clase social" y su vínculo con los perfiles de salud $[9,10]$. De esta forma, la DSS no solo alude a una propuesta metodológica para superar las limitaciones del causalismo lineal y el empirismo en salud, sometiendo a una evaluación crítica el empirismo propio de los análisis epidemiológicos y el predominio del enfoque cuantitativo de la investigación en salud, sino también a una propuesta ético-política para trabajar la reproducción social, que ocurre del plano general al singular, mediante la incorporación de la perspectiva dialéctica de los nexos entre sociedad-naturaleza-biología $[9,10]$.

En adición a los acontecimientos previamente descritos, es importante indicar que, pese a sus nexos y similitudes, la medicina social latinoamericana, la epi-

* Eduardo Cruz-Coke (1899-1974). Médico y político chileno que se desempeñó como ministro de Salud en su país en 1937 y 1938; impulsó políticas que fortalecieron el estado de bienestar en Chile y permitieron disminuir la morbilidad y mortalidad infantil. Para ampliar la información sobre la obra del doctor Cruz-Coke véase [15]

$\dagger$ Conferencia internacional sobre atención primaria en salud, realizada en Alma Ata (Kazajistán), en 1978. demiología crítica y la DSS corresponden a tres constructos diferentes, cada una con trayectorias históricas específicas. La medicina social latinoamericana constituye un movimiento de alta complejidad en el campo sanitario, cuyo comienzo se podría situar en el cierre del siglo XVIII y sus usos en epidemiología corresponden a la primera mitad del siglo Xx, con autores destacados como Allende (1930), Paredes (1938), Carrillo (1952), entre otros. La epidemiología crítica agrupa una diversidad de autores que cuestionaron los fundamentos de la epidemiología tradicional positivista, empírica y lineal, con diversas vertientes, como la etnosocial de los modos de vida de Almeida y Menéndez, la ecosocial de Krieger, la de la mercantilización y el desgaste de Laurell, y la de la Dss de Breilh. Así, esta última es una de las categorías centrales de la epidemiología crítica, cuyo autor con mayor continuidad y producción académica es Breilh [9,10,18-20].

La trayectoria histórica de la DSs ha permitido identificar al menos cuatro periodos de desarrollo conceptual y político: el primero se sitúa en la década de los setenta, llamado "periodo formativo", el cual se focalizó en la confrontación del paradigma lineal del riesgo; el segundo, el "periodo de diversificación", ocurrió durante la década de los ochenta y corresponde a la conjunción de intereses de los epidemiólogos críticos y el desarrollo teórico de la triple inequidad de clase, género y etnia; la tercera etapa se da en la década de los noventa y es el momento de la consolidación interdisciplinaria e intercultural de un nuevo sujeto de la epidemiología, como respuesta crítica a la epidemiología empírica y ecológica, y una crítica a la sociedad de mercado; la última etapa se despliega en el presente siglo, denominada "consolidación socionatural", con categorías complementarias a la DSS, como el metabolismo sociedad-naturaleza, las 4 "S" (sustentable, soberana, solidaria y saludable) de la vida, y la confrontación con la corriente de los determinantes sociales de la salud de la oms-ops. En los textos de Breilh en este último periodo, se hace alusión explícita al fundamento epistemológico de la DSs en el realismo crítico, para lo cual se toma como punto de partida la relectura de Thomas Kuhn y Pierre Bourdieu, autores cuyo uso en los textos de los representantes de la DSS es relativamente tardío o no se constata en sus manuscritos iniciales $[9,10,21]$.

\section{Algunas consideraciones útiles al análisis del estatus epistemológico de la DSS}

La Dss surge de la epidemiología crítica y toma conceptos importantes de las vertientes epistemológicas de Kuhn, con su visión del papel de la comunidad científica, y de Bourdieu, en tanto a la concepción de campo [9]. Asimismo, recoge algunos elementos de teorías del 
conflicto, de las teorías críticas y del materialismo histórico en ciencias sociales, para alinear su estatus epistemológico con postulados coherentes con el realismo crítico [11].

\section{Thomas Kuhn y la noción de comunidad científica}

Si bien Kuhn ha sido reconocido por el desarrollo del concepto de paradigma, para la DSs, la principal categoría epistemológica es la de "comunidad científica". La DSS se apoya en la obra de Kuhn para retomar varias características del pensamiento científico, como su perspectiva dialéctica, su naturaleza social y su característica de cambiante, temporal y revolucionario, esta última sustentada en las disputas entre las ideas innovadoras y las "normales" *-esto equivale a rescatar el abordaje sociopolítico y comunitario de la ciencia y sus dispositivos- [11].

Los aportes epistemológicos de Kuhn que sirven a la Dss giran en torno de su crítica al "realismo ingenuo", \$ a partir de un análisis del proceso dialéctico de las comunidades científicas. Para Kuhn, un paradigma cuenta con un núcleo de teorías y otro grupo secundario que funge como una suerte de protección frente a retos de otras teorías desconocidas; en esta estructura, la generación de conocimiento científico implica la participación de una comunidad (científica), con un tipo particular de relaciones en su interior, lo que evidencia que la ciencia también se configura como una producción social de un grupo social y sus valores, creencias, normas y relaciones, todos los cuales deberían explicitarse en todo tipo de análisis epistemológico [23].

\section{Pierre Bourdieu y su concepción de habitus y campo}

El pensamiento de Bourdieu se incorpora en la DSs desde el análisis que este realiza de "la teoría como un modus operandi que orienta y organiza la práctica científica" y las "capacidades creadoras, activas, inventivas del habitus", al tiempo que el lado activo del conocimiento científico se asume como "el capital de un sujeto trascendente", "de un agente en acción" (Bordieu, citado en $[9$, p. 13].

Bourdieu considera a la sociedad como relaciones de lucha y poder que se dan entre agentes (personas) o agencias (colectivos, grupos sociales, instituciones, organizaciones), con lo cual se toma distancia de algunas ontologías del individuo que asumen a las personas como sumatorias mecánicas en el concepto de población, como sujetos o actores, en cuyas epistemologías subyacen visiones de los individuos como entes estáticos, con poca posibilidad de cambio, sin historia, sin

$\$ \quad$ Periodos donde las comunidades científicas fortalecen un paradigma aceptado con base en verificaciones de sus postulados.

$\S \quad$ “Actitud filosófica desprovista de toda reflexión crítica acerca del conocimiento, que considera que las cosas son tal como las percibimos" [22]. cultura, atados a las estructuras o entes que desempeñan un papel predefinido [24,25]. Bourdieu propone que, aunque los agentes reproducen, en gran medida, lo que toman durante sus procesos de socialización, un agente es activo y su actuar puede modificar las condiciones de los grupos y los espacios sociales en donde discurre su vida, mediante la transformación de las reglas que definen dichos espacios, las mismas que privilegian a unos y marginan a otros. Esto tiene connotaciones importantes a la hora de trascender posturas epistemológicas que se alinean con el realismo ingenuo [24,25].

\section{Aportes desde la dialéctica histórica del materialismo}

El materialismo histórico/dialéctico, el cual es la base filosófica del marxismo, cumple un papel importante en la fundamentación epistemológica de la DSs (también ética, política, investigativa y de otras esferas que no se enuncian en este texto), al considerar como un aspecto determinante de las mediaciones de carácter sociohistórico y económico la relación sujeto-objeto, que funge como condición de posibilidad para la génesis teórica, en la medida en que el investigador no puede separarse de sus contextos, por lo que siempre es parte activa del fenómeno social que se estudia. En este marco confluyen diversas teorías marxianas (en las cuales es imposible desvincular lo social y lo político), neomarxianas, del conflicto y social crítica [26,27].

Desde esta perspectiva, el materialismo histórico/ dialéctico asumiría a la salud pública como entidad compleja y multidimensional, donde la realidad es un acontecimiento con su propio devenir histórico-crítico, que se mueve dialécticamente bajo la oposición y lucha de contrarios, y donde el sujeto es un ente activo, creador y transformador, y la investigación supone la integración de diferentes disciplinas, niveles de realidad y culturas [28].

En este orden de ideas, del materialismo marxiano se continúan tomando categorías determinantes para la configuración latinoamericana de la epidemiología crítica de inicios del siglo xxI y, en términos epistemológicos, reivindica la realidad en movimiento, que no niega el concepto de estructura, pero sí el estructuralismo (o falacia estructuralista), que anula las realidades y posibilidades de acción de los sujetos en los niveles particular y singular. El concepto de configuración, entendido como red de relaciones entre estructura, subjetividad y acción, trasciende la deducción, dado que, metodológicamente, se busca reconstruir la "totalidad concreta", esto es, reconstruir las configuraciones con sus componentes objetivos y subjetivos en momentos y situaciones históricas concretas [18,29].

En tal sentido, el dinamismo de la dialéctica sujeto-objeto (estructura-subjetividad) se involucra en un espacio de posibilidades para la acción. Así, el materialismo histórico dialéctico reconoce la interacción de 
niveles estructurales y subjetivos que determinan las opciones de acción de los sujetos, es decir, que el sujeto, como miembro de la estructura, tiene unos niveles de resistencia y autonomía que fortalecen la idea emancipatoria [29].

\section{Realismo crítico como eje epistemológico de la determinación social de la salud}

En coherencia con el cierre del apartado anterior, es importante precisar que la DSs tiene una fuerte raíz marxiana, que critica al positivismo y al realismo ingenuo, pero sin hacer explícita la noción de realismo crítico; de allí la importancia de remarcar que Breilh destaca el realismo crítico como fundamento epistemológico de la DSS en un texto del año 2013 [9,10]. Es oportuno entonces explicitar que el realismo crítico constituye un sistema filosófico, con un desarrollo independiente de la Dss, por lo que es muy probable que sus planteamientos no siempre se alineen; sin embargo, dada la reciente incorporación de esta postura filosófica en los textos de Breilh, principal representante de la DSs, es necesario hacer una descripción de sus principales características.

También es importante precisar que autores como Juan Carlos Eslava, en un artículo del año 2017, ya habían realizado un análisis epistemológico de la DSs; pero, en este caso, el autor no lo hace desde el realismo crítico, sino desde algunos planteamientos marxistas que fundamentan la medicina social latinoamericana [18]. A partir de una concepción de la esfera epistemológica como aquella que indica "la forma de entender el conocimiento científico y a la comprensión de la causalidad" $[18$, p. 399], Eslava destaca las siguientes temáticas marxianas que influenciaron la medicina social latinoamericana: el método basado en la transición de "lo concreto sensible al concreto de pensamiento" [18, p. 399], el pensamiento relacional y dialéctico, la superación del individualismo metodológico y "las categorías de producción, reproducción, mercancía, sujeto social, valor, trabajo y clase" [18, p. 399]. Estas categorías marxianas derivaron en el perfil epidemiológico de clase, analizado en las "dimensiones general, particular y singular" de la DSs, donde los modos de vida constituyen un concepto nuclear que ha sido operativizado de manera diversa por los representantes de la epidemiología crítica [18,19,30-32].

Lo anterior da cuenta de la novedad del análisis epistemológico de la Dss, que hace un uso explícito del realismo crítico, como postura que busca trascender el realismo ingenuo que predomina en el pensamiento salubrista de algunas comunidades académicas. En este orden de ideas, el realismo ingenuo, también denominado "realismo perceptivo directo", plantea que la percepción mediada por los sentidos es la fuente del conocimiento directo del mundo físico, esto es, la existencia y la naturaleza de los objetos se revela al sujeto sin intermediaciones [33].

En contraposición a esta ausencia de intermediación, la relación entre sujeto y objeto del realismo crítico plantea que la realidad es exterior a los sujetos, tanto en lo material como en lo inmaterial, diferencia central con el materialismo anterior a los desarrollos del marxismo, el cual le da mayor relevancia a la percepción sensorial y material de la naturaleza. El realismo crítico explica que el mundo real existe tanto si se teoriza sobre él como si no se hace, de manera que en ese mundo se presentan relaciones causales genuinas. Esto implica que los fenómenos observados son reales, pero no agotan la realidad, sino que son reflejo de efectos de muchas causas de mayor envergadura y complejidad [34].

Varios filósofos, como Alexander Callinicos, Karl Popper, Mario Bunge, Roy Bhaskar, entre otros, se han referido al realismo crítico, entre los cuales se destaca el último autor, por tener las obras más citadas en este tema. Bhaskar identifica los principales tipos de realismo: el predictivo, que alude a una existencia universal independiente, como rasgo de algunos objetos materiales; el perceptivo, que sitúa los objetos en términos espacio-temporales, y el científico, en el cual los objetos de la investigación son absolutos e independientes; todos estos realismos confluyen en la crítica al positivismo [35]. En tal sentido, se debe recordar que las posiciones predominantes en filosofía han sido el empirismo clásico, el idealismo trascendental y el realismo trascendental; en este último podría situarse el realismo crítico de Bhaskar, el cual contiene una esfera ontológica independiente del ser o "dimensión intransitiva del conocimiento", una esfera epistemológica o transitiva que, en coherencia con las formulaciones de Kuhn, es caracterizado por su contingencia social e histórica, lo que da una suerte de realismo trascendental ontológico, mezclado con un relativismo epistemológico [35-37].

El realismo crítico constituye un sistema de pensamiento, con una ontología específica, que pretende superar las limitaciones del positivismo y la hermenéutica; para ello, define la "realidad" como un constructo de tres dimensiones: 1) la empírica, que está constituida por las percepciones del observador; 2) la actual, que se manifiesta en acontecimientos espacio-temporales determinados, y 3) la denominada "verdadera" o "transfactual", configurada por poderes y restricciones perdurables que trascienden los acontecimientos o sucesos concretos. En esta postura epistemológica, el conocimiento se genera mediante el análisis de las estructuras y los mecanismos causales que permiten relacionar las tres dimensiones, y devela procesos de reproducción social, en una acepción de la realidad como totalidad multidimensional [34], es decir, no se trata de analizar estas dimensiones por sepa- 
rado, dado que el centro son sus interrelaciones, simultaneidad y superposición [36,38].

Lo transfactual es conocer los mecanismos causales que explican un fenómeno, que resultan ontológicamente intransitivos o independientes de la percepción de un sujeto; lo actual, son los fenómenos que se derivan de dichos mecanismos, y lo empírico configura el componente transitivo o dependiente de la percepción o de los prejuicios que permiten comprender la actualidad $[36,39,40]$.

En la línea de los planteamientos de Baskhar, las preguntas claves para orientar epistemológicamente la praxis científica serían: “¿Cómo procede el científico para desarrollar su actividad? ¿Qué busca el científico al desarrollar su actividad? o, más aún, ¿qué propiedades de la realidad son prerrequisito para que la práctica científica tenga sentido?" [39, p. 218]. A estas preguntas subyace una realidad ontológica, "la realidad existe independiente de nuestro conocimiento de ella" [39, p. 218], denotando con ello una distancia entre lo empírico y lo real, o planteado de otra forma, la imposibilidad de dar cuanta de la realidad mediante una única dimensión como la empírica (generalmente sesgada por preconceptos), al tiempo que se devela la importancia de la dimensión transfáctica, que permite conocer la causalidad de modo real y no como la observación de relaciones regulares [36,37,39-42].

En el realismo crítico se reconoce el hecho que los sistemas estudiados son abiertos, dada la imposibilidad de controlar las determinaciones de un evento; por ello, no es posible reducir la esfera epistemológica a lógicas inductivas o deductivas, dado que los fenómenos son reconocidos por su contingencia social, cultural e histórica, lo cual desmiente la falacia de la causalidad lineal o basada en las regularidades $[36,39,40]$.

Específicamente, en el campo social, Bhaskar alude la sociedad como "un conjunto articulado de estructuras generativas relativamente independientes y duraderas (es decir), como una totalidad compleja sujeta al cambio tanto en sus componentes como en sus interrelaciones" [37, p. 122]. De esta forma, las estructuras sociales ontológicamente son dependientes de los poderes que la conforman, existen de acuerdo con las visiones de mundo de los agentes, son variables en términos espaciotemporales y no son aprehensibles mediante el monismo metodológico, sino por medio de planteamientos relacionales [37].

Así, el conocimiento es una producción social en relación con saberes preexistentes (dimensión transitiva) y objetos cuyas acciones pueden ser independientes del investigador (dimensión intransitiva) [37,43]. En conjunción con ello, la realidad puede presentar diferentes tipologías, como la material o independiente de los sujetos, cuyo conocimiento es posible por la medición de los conceptos; la ideal o conceptual, cuya realidad se ve- rifica por la eficacia causal, la artefactual o mediada por tecnologías, y la social, donde confluyen las acciones; de esta forma, el realismo crítico es ontología multidimensional, que reconoce diferentes dominios de la realidad, con sus estructuras, agencia y mecanismos causales, que dan lugar a la reproducción o transformación social $[36,37,44]$.

En coherencia con lo expuesto, Breihl brinda una guía para materializar metodológicamente el realismo crítico, al indicar que "la epidemiología es pragmáticamente utópica; para serlo tiene que ser teórica y aplica$\mathrm{da}$, tiene que ser cuantitativa y cualitativa; tiene que ser académica y popular; tiene que ser filosófica, económica política, sociológica, antropológica, histórica, geográfica y ecológica; tiene que ser un brazo de ruptura y no de gobernanza de la salud pública" [9, p. 23]. Esto implica un llamado a que la epidemiología crítica y el modelo de la DSs aplique métodos mixtos, con una aproximación cuantitativa y otra cualitativa, con análisis sociohistórico y dialéctico, de modo tal que su combinación permita conocer la configuración de los procesos de determinación social de problemas de salud concretos en una población determinada $[9,10]$.

En este sentido, las exigencias epistemológicas de la Dss y su fundamento en el realismo crítico implican la necesidad de acoger métodos cuantitativos, cualitativos e históricos que permitan develar movimientos dialécticos en los diferentes niveles de la realidad (general, particular y singular). Así, la investigación en Dss debería dar cuenta del doble y opuesto movimiento entre la realidad socioeconómica y política que se reproduce del nivel general al singular, así como la resistencia y la movilización individual que, desde la dimensión singular, busca nuevas condiciones para generar una fuerza material colectiva [9].

\section{Conclusión}

La reflexión epistemológica de la DSs gira en torno del realismo crítico, en el cual han sido claves los aportes de Kuhn, Bourdieu, la dialéctica histórica del materialismo y la concreción realizada por Breilh.

Desde el realismo crítico se evidencia una suerte de "ingenuidad" epistemológica en el modelo de la Organización Mundial de la Salud; también demuestra que la diferencia de ambos enfoques no es reductible a una semántica o estilo de abordaje de lo social en salud, y pone de manifiesto retos operativos para la corriente latinoamericana.

\section{Fuente de financiación}

Estrategia de sostenibilidad de los grupos CoDI (Comité para el Desarrollo de la Investigación) Universidad de Antioquia 2018-2019. 


\section{Conflicto de intereses}

Los autores declaran no tener conflictos de intereses para la publicación del manuscrito.

\section{Declaración de responsabilidad}

Los puntos de vista expresados son responsabilidad de los autores y no de la institución en la que trabajan o de la fuente de financiación.

\section{Declaración de contribución por autores}

Juan Paulo Ruiz Taborda: participó en el diseño del artículo, búsqueda de literatura, análisis e interpretación de la información, revisión crítica de su contenido, aprobación de la versión final.

Luis Felipe Higuita Gutiérrez: participó en el diseño del artículo, búsqueda de literatura, análisis e interpretación de la información, revisión crítica de su contenido, aprobación de la versión final.

Jaiberth Antonio Cardona Arias: participó en el diseño del artículo, búsqueda de literatura, análisis e interpretación de la información, revisión crítica de su contenido, aprobación de la versión final.

\section{Referencias}

1. Organización Mundial de la Salud. Reducir las inequidades sanitarias actuando sobre los determinantes sociales de la salud [internet]; 2009 [citado 2020 mar. 10]. Disponible en: https://apps.who. int/iris/handle/10665/2497

2. Organización Mundial de la Salud. Determinantes sociales de la salud [internet]; 2008 [citado 2020 mar. 10]. Disponible en: http:// www.who.int/social_determinants/es/

3. Organización Panamericana de la Salud. Determinantes sociales de la salud en la región de las Américas [internet]; 2017 [citado 2020 mar. 10]. Disponible en: https://www. paho.org/salud-en-las-americas-2017/?post_type $=$ post_t es\&p=310\&lang=es\#: :text $=$ La $\% 20$ Organizaci $\% \mathrm{C} 3 \% \mathrm{~B} 3 \mathrm{n} \% 20$ Mundial\%20de\%20la,la\%20vida\%20cotidiana\%20(\%201\%20)

4. Programa de las Naciones Unidas para el Desarrollo. Objetivos de desarrollo del milenio [internet]; 2020 [citado 2020 oct. 22]. Disponible en: https://www.undp.org/content/undp/es/home/sdgoverview/mdg_goals.html

5. Organización Panamericana de la Salud. Organización Mundial de la Salud. Estrategia para el acceso universal a la salud y la cobertura universal de salud. 53. ${ }^{\circ}$ Consejo Directivo, $66 .^{\mathrm{a}}$ sesión del Comité Regional de la oms para las Américas, Washington [internet]; 2014 [citado 2020 mar. 10]. Disponible en: https://www. paho.org/hq/index.php?option $=$ com_docman\&task $=$ doc_downlo ad\&gid $=27273 \&$ Itemid $=270 \&$ lang $=$ es

6. Solar O, Irwin A. A Conceptual Framework for Action on the Social Determinants of Health. Ginebra: Organización Mundial de la Salud [internet]; 2007 [citado 2020 may. 20]. Disponible en: http://www.who.int/sdhconference/resources/ConceptualframeworkforactiononSDH_eng.pdf
7. Mackenbach JP. Political determinants of health. Eur J Public Health. 2014;24(1):2. DoI: https://doi.org/10.1093/eurpub/ckt183

8. Granda J. Determinación social de la muerte materna en el cantón Ibarra, Imbabura, Ecuador. [Tesis de maestría]. [Quito]: Universidad Andina Simón Bolívar; 2018.

9. Breilh J. La determinación social de la salud como herramienta de transformación hacia una nueva salud pública (salud colectiva). Rev. Fac. Nac. Salud Pública [internet]. 2013 [citado 2020 oct. 22]; 31(Supl. 1): 13-27. Disponible en: http:// www.scielo.org.co/scielo.php?script $=$ sci_arttext\&pid $=\mathrm{S} 0120$ 386X2013000400002\&lng=en

10. Breilh J. Epidemiología crítica latinoamericana: raíces, desarrollos recientes y ruptura metodológica. En: Morales C, Eslava JC. Tras las huellas de la determinación. Memorias del Seminario Interuniversitario de Determinación Social de la Salud. Facultad de Medicina, Facultad de Odontología, Universidad Nacional de Colombia. Bogotá; 2015. pp. 20-85.

11. Morales C, Borde E, et al. ¿Determinación social o determinantes sociales? Diferencias conceptuales e implicaciones praxiológicas. Rev Salud Pública [internet]. 2013 [citado 2020 oct. 22]; 15(6):797-808. Disponible en: http://www. scielo.org.co/scielo.php?script $=$ sci_arttext\&pid $=$ S012400642013000600001\&lng=en

12. Peñaranda F. Salud pública y justicia social en el marco del debate determinantes - determinación social de la salud. Rev. Fac. Nac. Salud Pública [internet]; 2013 [citado 2020 oct. 22]; 31(Supl. 1):91-102. Disponible en: http://www.scielo.org.co/pdf/rfnsp/ v31s1/v31s1a11.pdf

13. Anderson M. La medicina y la salud pública al final del imperio, reflexiones a partir del libro de Howard Waitzkin. Medicina Social [internet]. 2012 [citado 2020 oct. 22]; 7(1):1-4. Disponible en: https://www.medicinasocial.info/index.php/medicinasocial/ article/view/658/1273

14. Rojas Ochoa F. La Salud Pública Revolucionaria Cubana en su aniversario 50. Rev Cuba Salud Pública [internet]. 2009 [citado 2020 oct. 22]; 35(1):1-15. Disponible en: http://scielo.sld.cu/scielo.php?script=sci_arttext\&pid=S0864-34662009000100003

15. Huneeus C, Lanas MP. Ciencia política e historia: Eduardo CruzCoke y el estado de bienestar en Chile, 1937-1938. Historia (Santiago). 2002;35:151-86. DoI: http://dx.doi.org/10.4067/S071771942002003500007

16. Apráez Ippolito G. La medicina social y las experiencias de atención primaria de salud (APS) en Latinoamérica: historia con igual raíz. Polis Revista Latinoamericana [internet]. 2010 [citado 2020 may. 20]; 9(27): Disponible en: https://www.redalyc.org/articulo. oa? id=30515709017

17. Restrepo-Espinosa M. Orígenes y trayectorias de la atención primaria en salud. Desde Kazajistán a América. Rev Nova et Vetera. 2016;22(2)

18. Eslava-Castañeda J. Pensando la determinación social del proceso salud-enfermedad. Rev. Salud Pública. 2017;19(3):396-403. DoI: https://doi.org/10.15446/rsap.v19n3.68467

19. Breilh J. Epidemiología crítica: ciencia emancipadora e interculturalidad. Buenos Aires: Lugar Editorial; 2003.

20. Eslava JC. Explorando los inicios de la labor crítica de un pionero de la medicina social latinoamericana. En: Morales C, Eslava JC. Tras las huellas de la determinación. Memorias del Seminario Interuniversitario de Determinación Social de la Salud. Facultad de Medicina, Facultad de Odontología, Universidad Nacional de Colombia. Bogotá; 2015. pp. 117-28.

21. Breilh. Las tres "S" de la determinación de la vida: 10 tesis hacia una visión crítica de la determinación social de la vida y la salud. 
En: Centro Brasileiro de Estudos de Saúde (CEBES), Rediscutindo a quesdo da determinando social da saúde. Salvador, Brasil: CEBES; 2010. pp. 87-125.

22. Oxford English and Spanish Dictionary. Realismo ingenuo [internet]; 2020 [citado 2020 oct. 22]. Disponible en: https://www. lexico.com/es/definicion/realismo_ingenuo

23. Kuhn T. La estructura de las revoluciones científicas. México: Fondo de Cultura Económica; 2013.

24. Bourdieu P. Sobre el poder simbólico [internet]; 2000 [citado 2020 may. 20]. Disponible en: https://sociologiac.net/biblio/Bourdieu_SobrePoderSimbolico.pdf.

25. Chapela M. Campo y capital en la sociología de Pierre Bourdieu como aporte a la comprensión de la salud humana [internet]; 2014 [citado 2020 may. 20]. Disponible en: http://proinapsa.uis.edu.co/ redcups/Biblioteca/Promocion\%20salud\%20critica/ChapelaBourdieuPDFAgo25,14.pdf

26. Valencia M. Aportes de los nuevos enfoques para la conformación de la salud pública alternativa. Rev. Fac. Nac. Salud Pública [internet]. 2011 [citado 2020 oct. 22]; 29(1):85-93. Disponible en: http://www.scielo.org.co/scielo.php?script=sci arttext\&pid=S0120-386X2011000100010\&lng=en

27. Ruiz C. Crítica y dialéctica. La cuestión del método en el pensamiento de Marx. [Tesis de doctorado]. [Madrid:] Facultad de Filosofía, Universidad Complutense de Madrid; 2010.

28. Linares Pérez N. Aplicación de los enfoques de salud de la población y los determinantes sociales en Cuba. Rev Cuba Salud Publica [internet]. 2015 [citado 2020 oct. 22]; 41(1):94-114. Disponible en: http://scielo.sld.cu/scielo.php?script=sci_arttext\&pid $=$ S0864-34662015000100009

29. Garza Toledo E de la. La metodología marxista y el configuracionismo latinoamericano. En: Garza Toledo E de la, Leyva G. Tratado de metodología de las ciencias sociales: perspectivas actuales. México: Fondo de Cultura Económica; 2014. pp. 229-55.

30. Mendoza LM, Jarillo E. Determinación y causalidad en salud colectiva. Algunas consideraciones en torno a sus fundamentos epistemológicos. Ciência \& Saúde Coletiva, 2011; 16 (Supl. 1): 847-54. DoI: https://doi.org/10.1590/S1413-81232011000700016

31. Castellanos PL. Los modelos explicativos del proceso salud-enfermedad: los determinantes sociales. En: Martínez Navarro et al. Salud pública. Madrid: McGraw Hill, Interamericana; 1998. pp. 81-102.

32. Almeida-Filho A. La ciencia tímida. Ensayos de deconstrucción de la epidemiología. Buenos Aires: Lugar Editorial; 2000.
33. González Luna EM. La vuelta a la ingenuidad: un comentario sobre el realismo natural de Hilary Putnam. Rev Valencia Estud Filos y Let. 2009;(3):103-130. Dor: https://doi.org/10.15174/ rv.v0i3.402

34. Hausman D. El realismo crítico y las teorías de sistemas abiertos. Argumentos de Razón Técnica [internet]. 2000 [citado 2020 oct. 22]; (3):61-92. Disponible en: http://institucional.us.es/revistas/ argumentos/3/art_2.pdf

35. Hamlin C. Realismo crítico: um programa de pesquisa para as Ciências Sociais. Dados. 2000;43(2). Dor: https://dx.doi. org/10.1590/S0011-52582000000200006

36. Bhaskar R. Philosophy and scientific realism. En: Bhaskar R, Archer M, Collier A, et al., editores. Critical Realism: Essential Readings. Londres, Nueva York: Routledge; 1998. pp. 16-47.

37. Bhaskar R. Una teoría realista de la ciencia. Niterói: UFF, 2000.

38. Costa Delaine M, Magalhães R. Realismo crítico e desigualdades sociais: considerações a partir de uma pesquisa avaliativa. Ciênc. Saúde Coletiva [internet]. 2020 [citado 2020 oct. 22]; 25(5):177988. Disponible en: http://www.cienciaesaudecoletiva.com.br/ artigos/realismo-critico-e-desigualdades-sociais-consideracoes-apartir-de-uma-pesquisa-avaliativa/17420?id=17420

39. Parra Heredia J. Realismo crítico: una alternativa en el análisis social. Sociedad y Economía [internet]. 2016 [citado 2020 oct. 22]; (31):215-38. Disponible en: http://www.scielo.org. co/scielo.php?pid=S1657-63572016000200010\&script $=$ sci abstract\&tlng=es

40. Danermark B, Mats E, et al. Explaining Society: Critical Realism in the Social Sciences. Nueva York: Psychology Press; 2002.

41. Vandenberghe F. What's Critical about Critical Realism? Essays in Reconstructive Social Theory. Londres, Nueva York: Routledge; 2014.

42. Vandenberghe F. The normative foundations of critical realism: A comment on Dave Elder-Vass and Leigh Price. Journal of Critical Realism. 2019;18(3):319-36. DoI: https://doi.org/10.1080/147674 30.2019 .1638607

43. Reed M. Reflections on the "Realist Turn" in organization and management studies. Journal of Management Studies. 2005;42(8):1621-44. DoI: https://doi.org/10.1111/j.14676486.2005.00559.x

44. Borges A, Brito M, et al. Contribuições do diálogo entre o realismo crítico e o construcionismo social para os estudos organizacionais. Cadernos EBAPE.BR. 2016;14(2):391-405. DOI: https://doi. org/10.1590/1679-395122299 